\title{
Emergence of VIM-6 metallo-beta-lactamase-producing Alcaligenes faecalis clinical isolates in a hospital in India
}

\author{
Atul Khajuria, Ashok Kumar Praharaj, Mahadevan Kumar, Naveen Grover \\ Department of Microbiology, Armed Forces Medical College, Pune, India
}

Key words: Alcaligenes faecalis; multidrug resistant; carbapenems; bla $_{\mathrm{VIM}-6}$

J Infect Dev Ctries 2013; 7(6):494-496. doi:10.3855/jidc.3556

(Received 17 March 2013 - Accepted 07 April 2013)

Copyright (C) 2013 Khajuria et al. This is an open-access article distributed under the Creative Commons Attribution License, which permits unrestricted use, distribution, and reproduction in any medium, provided the original work is properly cited.

\section{Background}

Alcaligenes faecalis (Affaecalis) is a Gramnegative, rod-shaped, motile, oxidase, catalase and citrate positive obligate aerobe that is commonly found in the environment. A. faecalis is the most frequently isolated member of family Alcaligenaceae in the clinical laboratory. Infections caused by $A$. faecalis are opportunistic and are acquired from moist items such as nebulizers, respirators, and lavage fluid [1]. It has been reported to cause peritonitis, urinary tract infection (UTI), bacteremia and acute otitis media $[1,2]$. Although presence of PER-1 and TEM-21 Extended Spectrum Beta-Lactamases (ESBL) have been reported in clinical isolates of A. faecalis $[3,4]$, carbapenem resistance has not yet been reported. This study aimed to investigate the mechanism of carbapenem resistance among clinical isolates of $A$. faecalis recovered from hospitalized patients in a tertiary care centre in Pune, India.

\section{The study}

During the study period from January 2012 to December 2012, a total of 15 clinical isolates of $A$. faecalis were recovered from different clinical specimens such as urine, pus, blood and body fluids, from patients admitted to the medical and surgical intensive care units in a 1,000-bed tertiary care hospital in Pune, India. Bacterial identification was performed by routine conventional microbial culture and biochemical tests [5]. The organisms were identified up to the species level using VITEK-GNI cards (bioMérieux, Marcy l'Etoile, France). The antibiotic sensitivity test was performed by the standard Kirby Bauer disc diffusion method per the guidelines of the Clinical Laboratory Standards Institute (CLSI), using commercially available antibiotic discs (Hi Media, Mumbai, India) on Mueller Hinton agar plates [6]. Minimum inhibitory concentrations (MIC) of antibiotics were determined by VITEK-2 against imipenem, meropenem, ticarcillin, amikacin, gentamicin, tobramycin, ciprofloxacin, levofloxacin, moxifloxacin, tigecycline, trimethoprim/sulfamethoxazole, ampicillin/sulbactam, piperacillin/tazobactam, cefoperazone/sulbactam, cefepime, tetracycline, ceftazidime, ceftriaxone and colistin. E. coli ATCC 25922 and $P$. aeruginosa ATCC 27853 strains were used for quality control. Seven of these isolates were found to be carbapenem resistant according to CLSI breakpoints. All the isolates exhibiting reduced susceptibility to any of the carbapenems (meropenem and imipenem) by disc diffusion were screened for the production of carbapenemase by the Modified Hodge Test (MHT). Clover leaf type indentation by the isolates was interpreted as positive MHT. All seven isolates showed a positive MHT. Screening for metallo-betalactamase (MBL) production was performed on these isolates by the E-test MBL IP/IPI method and all of them were found to be positive for MBL. DNA was extracted using the spin column method (QIAGEN; $\mathrm{GmbH}$, Hilden, Germany) per the manufacturer's instructions. PCR amplification for detection of Ambler class B MBLs $b l a_{\mathrm{IMP}}, b l a_{\mathrm{VIM}}, b l a_{\mathrm{SPM}}, b l a_{\mathrm{GIM}}$, $b l a_{\mathrm{SIM}}$ and $b l a_{\mathrm{NDM}-1}$ and Ambler class D bla $a_{\mathrm{OXA}-23}$, $b l a_{\text {OXA-24 }}$ and $b l a_{\text {OXA48 }}$ was carried out on the isolates in a Gene Amp 9700 PCR System (Applied Biosystems, Singapore). Primers used for the PCR were as described earlier [7]. These isolates were 
found to be positive for $b l a_{\mathrm{VIM}-6}$ by polymerase chain reaction. The amplicons were purified using QIAquick PCR purification kit (QIAGEN; GmbH, Hilden, Germany) and sequenced with the ABI 3730XL capillary sequencer (Applied Biosystems, Foster City, CA, USA). The sequence of the bla $a_{\mathrm{VIM}-6}$ gene showed $100 \%$ identity with previously reported genes.

MIC values of these seven isolates are shown in the Table. The clonality of $A$. faecalis was confirmed by REP-PCR and ERIC PCR; the resulting banding pattern confirmed that two clones were circulating in the hospital environment [8].

The development and spread of resistance is a complex process that is influenced by selective pressure, pre-existence of resistance genes, and the use of infection control measures. Achromobacter xylosoxidans (previously Alcaligenes xylosoxidans) is recognized as a clinically significant nosocomial pathogen with its tendency to contaminate fluids. Many outbreaks of this organism have been reported earlier. The presence of metallo-beta-lactamases, such as IMP-1, VIM-1, or VIM-2, has been reported for $A$. xylosoxidans, consequently leading to high-level resistance to carbapenems [9]. Carbapenem resistance has not yet been reported for $A$. faecalis. The importance of the microbiology laboratory cannot be overemphasized in the detection and control of the spread of carbapenemase-resistant organisms. It is therefore imperative that the laboratory should have measures in place for the detection of carbapenemases.

The nucleotide sequence data of the bla $a_{\mathrm{VIM}-6}$ gene from the three clinical isolates of $A$. faecalis reported in the present study have been assigned GenBank nucleotide numbers KC493967, KC493968 and KC493969.

Table. Antibiotic susceptibilities of bla ${ }_{\mathrm{VIM}-6}$ positive Alcaligenes faecalis (mg/L)

\begin{tabular}{cccccccc}
\hline Antibiotics/ Isolate No. & $\mathrm{AF} / 1860$ & $\mathrm{AF} / 1991$ & $\mathrm{AF} / 2260$ & $\mathrm{AF} / 2076$ & $\mathrm{AF} / 2077$ & $\mathrm{AF} / 2089$ & $\mathrm{AF} / 2173$ \\
\hline IPM & 16 & 16 & 16 & 32 & 32 & 16 & 16 \\
MEM & 16 & 16 & 16 & 32 & 32 & 16 & 16 \\
AMK & 64 & 64 & 64 & 64 & 64 & 64 & 64 \\
GEN & 16 & 16 & 16 & 16 & 16 & 16 & 16 \\
TOB & 16 & 16 & 16 & 16 & 16 & 16 & 16 \\
CIP & 4 & 4 & 4 & 4 & 4 & 4 & 4 \\
LVX & 4 & 4 & 4 & 8 & 8 & 4 & 4 \\
TIC & 128 & 128 & 128 & 128 & 128 & 128 & 128 \\
TGC & 2 & 2 & 2 & 4 & 4 & 2 & 2 \\
SXT & 320 & 320 & 320 & 320 & 320 & 320 & 320 \\
SAM & 32 & 32 & 32 & 32 & 32 & 32 & 32 \\
TZP & 128 & 128 & 128 & 128 & 128 & 128 & 128 \\
FEP & 64 & 64 & 64 & 64 & 64 & 64 & 64 \\
SFP & 64 & 64 & 64 & 64 & 64 & 64 & 64 \\
CRO & 64 & 64 & 64 & 64 & 64 & 64 & 64 \\
CAZ & 64 & 64 & 64 & 64 & 64 & 64 & 64 \\
TET & 4 & 4 & 4 & 16 & 16 & 4 & 4 \\
CST & 0.5 & 0.5 & 0.5 & 0.5 & 0.5 & 0.5 & 0.5 \\
\hline
\end{tabular}

IPM = imipenem; MEM = meropenem; AMK = amikacin; GEN = gentamicin; TOB = tobramycin; CIP = ciprofloxacin;

$\mathrm{LVX}=$ levofloxacin; $\mathrm{TIC}=$ ticarcillin; $\mathrm{TGC}=$ tigecycline; $\mathrm{SXT}=$ trimethoprim $/$ sulfamethoxazole; $\mathrm{SAM}=$ ampicillin/sulbactam;

$\mathrm{TZP}=$ piperacillin/tazobactam; SFP = cefoperazone/sulbactam; FEP = cefepime; TET = tetracycline; CAZ = ceftazidime;

$\mathrm{CRO}=$ ceftriaxone CST $=$ colistin 


\section{References}

1. Winn W Jr, Allen S, Janda W, Koneman E, Procop G, Schreckenberger P, Woods G, editors (2006) The Nonfermentative Gram-negative bacilli, Chapter 7. In: Koneman's Color Atlas and Textbook of Diagnostic Microbiology, $6^{\text {th }}$ ed. Baltimore: Lippincott Williams and Wilkins Company 303391.

2. Kavuncuoglu F, Unal A, Oguzhan N, Tokgoz B, Oymak O, Utas C (2010) First reported case of Alcaligenes faecalis peritonitis. Perit Dial Int 30: 118-119.

3. Pereira M, Perilli M, Mantengoli E Luzzaro F, Toniolo A, Rossolini GM, Amicosante G (2000) PER-1 extended spectrumb-lactamase production in an Alcaligenes faecalis clinical isolate resistant to expanded-spectrum cephalosporins and monobactams from a hospital in Northern Italy. Microb Drug Resist 6: 85-90.

4. Dubois V, Arpin C, Coulange L, André C, Noury P, Quentin C (2006) TEM-21 extended-spectrum beta-lactamase in a clinical isolate of Alcaligenes faecalis from a nursing home. $\mathrm{J}$ Antimicrob Chemother 57: 368-369.

5. Collee JG, Miles RS, Wan B (1996) Tests for the identification of bacteria. In: Collee JG, Fraser AG, Marmion BP, Simmons A, editors. Mackie and McCartney Practical Medical Microbiology. 14th ed. Edinburgh: Churchill Livingstone: 131-150.
6. Clinical and Laboratory Standards Institute (2012) Performance Standards for Antimicrobial Susceptibility Testing: twenty second Informational Supplement M100-S22. CLSI, Wayne, PA, USA.

7. Khajuria A, Praharaj AK, Grover N, Kumar M (2013) First Report of blaNDM-1 in Raoultella ornithinolytica. Antimicrob Agents Chemother 57: 1092-1093.

8. Versalovic J, Koeuth T, Lupski JR (1991) Distribution of repetitive DNA sequences in eubacteria and application to fingerprinting of bacterial genomes. Nucleic Acids Res 19: 6823-6831.

9. Doi Y, Poirel L, Paterson DL, Nordmann P (2008) Characterization of a naturally occurring class D betalactamase from Achromobacter xylosoxidans. Antimicrob Agents Chemother 52:1952-1956.

\section{Corresponding author}

Atul Khajuria

Department of Microbiology

Armed Forces Medical College

Pune 411040, India

Telephone: 02026026058

Email: atulafmc@gmail.com

Conflict of interests: No conflict of interests is declared. 